\title{
Corrosion Inhibition of Aluminium in Gas and Acid Media by Some Chalcone-Based N-(3-Aminopropyl)Imidazoles: TD-DFT-Based FMO, Conceptual DFT, QTAIM and EDA Studies
}

\author{
Fritzgerald Kogge Bine1, Stanley Numbonui Tasheh1, Nyiang Kennet Nkungli2, \\ Julius Numbonui Ghogomu ${ }^{1,2^{*}}$
}

${ }^{1}$ Research Unit of Noxious Chemistry and Environmental Engineering, Department of Chemistry, Faculty of Science, University of Dschang, Dschang, Cameroon

${ }^{2}$ Department of Chemistry, Faculty of Science, The University of Bamenda, Bamenda, Cameroon

Email: *ghogsjuju@hotmail.com

How to cite this paper: Bine, F.K., Tasheh, S.N., Nkungli, N.K. and Ghogomu, J.N. (2021) Corrosion Inhibition of Aluminium in Gas and Acid Media by Some Chalcone-Based N-(3-Aminopropyl)Imidazoles: TD-DFT-Based FMO, Conceptual DFT, QTAIM and EDA Studies. Computational Chemistry, 9, 37-63.

https://doi.org/10.4236/cc.2021.91003

Received: November 5, 2020

Accepted: January 2, 2021

Published: January 5, 2021

\section{Copyright $\odot 2021$ by author(s) and} Scientific Research Publishing Inc. This work is licensed under the Creative Commons Attribution International License (CC BY 4.0).

http://creativecommons.org/licenses/by/4.0/

\begin{abstract}
The efficacy and mode of action of five chalcone-based imidazole derivatives as corrosion inhibitors of aluminium metal in gas-phase and acidic medium have been investigated herein via quantum chemical calculations. Dispersion-corrected DFT (DFT-D3) and time-dependent DFT (TD-DFT) calculations were performed at PBE0/def2-TZVP//PBEh-3c and CAM-B3LYP/def2TZVP levels of theory, respectively. Conceptual DFT, the quantum theory of atoms-in-molecules (QTAIM) and local energy decomposition (LED) analyses have been performed. The LED analysis was performed at the coupled-cluster singles and doubles with perturbative triples $(\mathrm{CCSD}(\mathrm{T}))$ /def2-SVP level of theory. Frontier molecular orbital energy gaps calculated using the TD-DFT method are found to lie in the range $3.574-4.444 \mathrm{eV}$, indicative of good adsorption and corrosion inhibition efficacies of the investigated molecules. The interactions between aluminium and the inhibitor molecules studied are found to be energetically favorable, owing to negative computed interaction energy values. Furthermore, QTAIM analysis revealed metal-carbon, metal-oxygen and metal-nitrogen interactions in the inhibitor-aluminium complexes, which are predominantly electrostatic in character, according to LED analysis results. Calculated proton affinities (PAs) have revealed the anticorrosion potentials of the investigated inhibitors in acidic medium, with a noticeable dependency on temperature within the range $273.15-343.15 \mathrm{~K}$.
\end{abstract}




\section{Keywords}

Density Functional Theory, Anticorrosion, Inhibitor-Aluminium

Interactions, Quantum Theory of Atoms-in-Molecules, Local Energy

Decomposition Analysis

\section{Introduction}

In recent times, considerable research attention is focused on the design and synthesis of organic molecules as corrosion inhibitors for metals and their alloys. Indeed, corrosion inhibition plays a crucial role in several industrial applications, since the deterioration of metals over time has a negative impact on their technical, economic, ecological and aesthetic quality standards [1]. It is well known that organic compounds containing heteroatoms (such as oxygen, nitrogen and sulfur) and conjugated double bonds are effective corrosion inhibitors [2]. Upon adsorption on a metal surface, electrons are transferred from an organic inhibitor to vacant orbitals of a metal, leading to covalent and/or non-covalent interactions (closed shell interactions) between the metal and the inhibitor. Based on several studies [3] [4] [5], it has been found that the adsorption of organic inhibitors on metal surfaces is mainly dependent on physicochemical properties of the inhibitors, including: the type of functional groups present, the electron density at the donor atoms (typically $\mathrm{S}, \mathrm{N}$ and $\mathrm{O}$ ), the molecular size, the mode of adsorption, the formation of organo-metal complexes, and the estimated area of the inhibitor on the metallic surface (degree of surface coverage). Furthermore, the need to choose corrosion inhibitors that are nontoxic and eco-friendly cannot be underestimated. In this regard, chalcones and their derivatives have drawn much interest, owing to their high tolerance in the human body (especially when complexed with metals) [6] [7]. Moreover, environmental pollution by inorganic corrosion inhibitors has prompted the search for green organic corrosion inhibitors that are biodegradable and do not contain heavy metals or other toxic constituents.

Aluminium is one of the most extensively used metals in the world today, but it undergoes deterioration when exposed to a corrosive environment (such as an acidic medium), in spite of its ability to form a thin protective oxide film on its surface. This has warranted the constant search for more effective and eco-friendly inhibitors of aluminium corrosion in a bid to increase the shelf life of aluminium-based materials [1].

Accordingly, the main objective of this work was to investigate the corrosion inhibitive capabilities, as well as the inhibitory modes of action, of some chalcone-based N-(3-aminopropyl) imidazole derivatives against the corrosion of aluminium in gas-phase and acidic medium via theoretical calculations using the density functional theory (DFT), time dependent density functional theory (TD-DFT) and coupled-cluster singles and doubles with perturbative triples 
(CCSD (T)) methods. In addition, the inhibitor-aluminium interactions have been elucidated herein in order to provide further insights into the corrosion inhibition mechanisms of the compounds studied.

It is interesting to note that theoretical methods such as those mentioned above have been successfully used in previous works to study the corrosion inhibition efficiency, molecular structures and electronic properties of organic corrosion inhibitors [8]-[14] for various metals, reason why such methods were employed in the present work. The DFT has been principally used in this study because many useful molecular parameters and descriptors in characterizing the corrosion inhibitor effectiveness on metal surfaces have been formulated within the framework of conceptual DFT [15].

To achieve the aforementioned objective of this work, the following five chalcone-based N-(3-aminopropyl)imidazoles have been studied (see Figure 1 for their molecular structures):

2-[1-(3-(1H-imidazol-1-yl)propylimino)-3-(phenylallyl)]phenol (HL1),

2-[1-(3-(1H-imidazol-1-yl)propylimino)-3-4-nitrophenylallyl]phenol (HL2),

2-[1-(3-(1H-imidazol-1-yl)propylimino)-3-4-methoxyphenylallyl]phenol (HL3),

2-[1-(3-(1H-imidazol-1-yl)propylimino)-3-4-hydroxyphenylallyl]phenol (HL4),

2-[1-(3-(1H-imidazol-1-yl)propylimino)-3-4-fluorophenylallyl]phenol (HL5).

This study for the first time, not only illustrates possible inhibitor-aluminium interactions, but also reveals quantitative insights into the inhibitor-aluminium interactions through the well-established quantum theory of atoms in molecules (QTAIM) and energy decomposition analysis (EDA) methods. To the best of our knowledge, the inhibitor-aluminium interactions involving the organic inhibitors currently studied have not been investigated both experimentally and theoretically. This study therefore provides useful data for subsequent experimental investigations on corrosion inhibition of aluminium by chalcone-based $\mathrm{N}$-(3-aminopropyl) imidazoles.
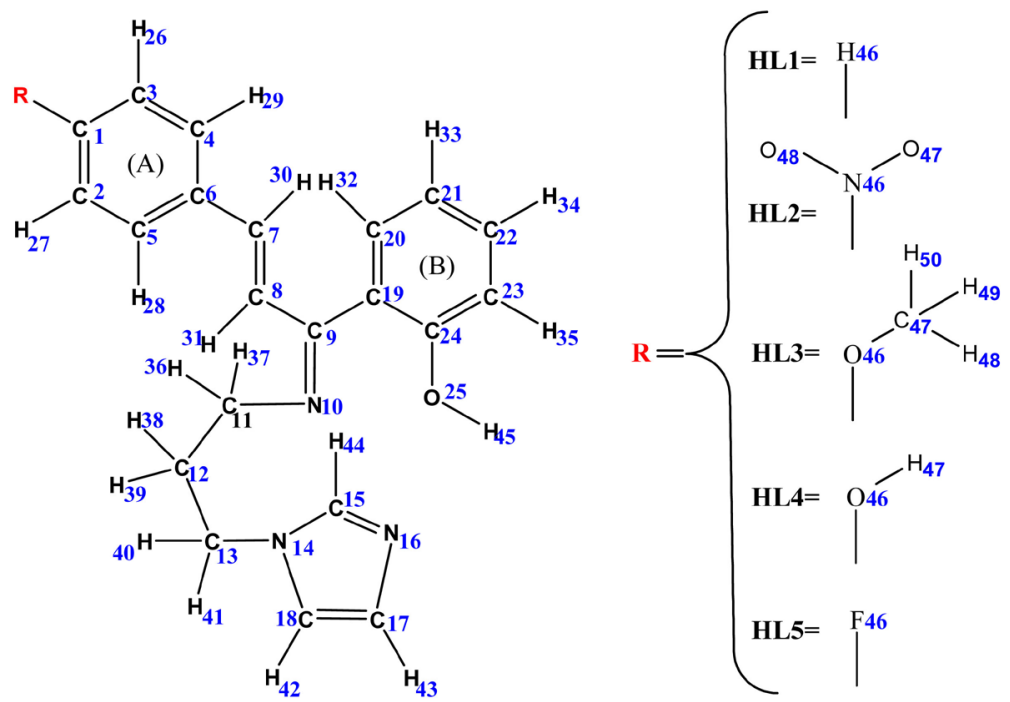

Figure 1. Schematic structure and numbering scheme of the studied inhibitors. 


\section{Computational Details and Theory}

Theoretical calculations were carried out with the ORCA 4.1.0 and 4.2.0 computational packages [16]. The ORCA 4.2.0 package was only used for the calculations in solvent medium. The input files were prepared with the Avogadro 1.1.1 visualization software [17]. Geometry optimization and frequency calculations in both gas and solvent phases were carried out using the low-cost composite electronic structure approach dubbed PBEh-3c [18]. It is worth indicating that the PBEh-3c method is a highly efficient electronic structure approach for geometry optimization calculations and interaction energy computation for non-covalent complexes [18]. Here, the global hybrid variant of the Perdew-Burke-Ernzerhof (PBE) functional that incorporates a relatively large amount of non-local exact Hartree-Fock exchange (42\%) is employed, along with a valence-double-zeta gaussian atomic orbital basis set (def2-mSVP) [18]. Also, in this study the composite def2-mSVP basis set is made up of the following standard basis sets: def2-SV (P) ( $\alpha$ scaled by 1.2) for hydrogen, def2-SV (P) for aluminium, the Ahlrich's DZ basis including polarization from $6-31 G^{*}$ for carbon, nitrogen and oxygen. Additionally, the PBEh-3c approach accounts for short-range basis set incompleteness (SRB), basis set superposition errors (BSSE) and long-range London dispersion effects. The latter two corrections are incorporated via the $\mathrm{gCP}$ and $\mathrm{D} 3$ schemes, respectively, where gCP stands for the molecular geometrical counterpoise correction which takes care of BSSE [19], D3 represents one of the latest Grimme's empirical dispersion-correction schemes [20]. Reliable and stable structures (global minima on the PES) were confirmed via the calculation of vibrational frequencies for all optimized structures at the PBEh-3c level of theory, which revealed the absence of any imaginary frequencies.

Based on the optimized gas-phase inhibitor-aluminium complexes, energy decomposition analyses via the local energy decomposition (LED) approach were carried out using the CCSD (T) method. To speed up these calculations, the domain-based local pair natural orbital (DLPNO) approximation was used, leading to the DLPNO-CCSD (T) approach. It is worthy of note that the open-shell DLPNO-CCSD (T) algorithm [21] [22], as implemented in the ORCA 4.1.0 package, was used to perform the LED calculations at the DLPNO-CCSD (T)/ def2-SVP level of theory, based on the "NormalPNO" approach.

In order to calculate ionization energies and electron affinities within the conceptual DFT framework, single point calculations on the PBEh-3c optimized geometries were performed at PBE0-D3/def2-TZVP level of theory. To speed up these calculations, albeit incurring a minimal loss of accuracy, the resolution-of-the-identity (RI-J) approximation [23] was used, along with the chain-of-spheres (COSX) approximation [24] (leading to the RIJCOSX approximation). The numerical quadrature integration grid5 was used in these calculations. In the implementation of conceptual DFT, global reactivity descriptors (GRDs) such as electronegativity $(\chi)$, global hardness $(\eta)$, global softness $(S)$ 
and electrophilicity index $(\omega)$ have been calculated according to Equations (1)-(6) and Equation (10):

$$
\begin{gathered}
\chi=-\mu=-\left(\frac{\partial E}{\partial N}\right)_{v(r)}=\frac{1}{2}(I P+E A) \\
\eta=\frac{1}{2}\left(\frac{\partial^{2} E}{\partial N^{2}}\right)_{v(r)}=\frac{1}{2}\left(\frac{\partial \mu}{\partial N}\right)_{v(r)}=\frac{1}{2}(I P-E A) \\
S=\frac{1}{2 \eta} \\
\omega=\frac{\mu^{2}}{2 \eta}
\end{gathered}
$$

In Equations (1)-(4), $\mu$ and $v(r)$ respectively represent the chemical and external potentials of an $N$-electron system with total energy $E$. IP and $E A$ are the first vertical ionization potential and electronic affinity of the system, respectively. Values of $I P$ and $E A$ are calculated according to the following Equations (5) and (6).

$$
\begin{gathered}
I P=E_{N-1}-E_{N} \\
E A=E_{N}-E_{N+1}
\end{gathered}
$$

where $E_{N}, E_{N-1}$ and $E_{N+1}$ are single point (SP) energies of the neutral, cationic and anionic forms of the $N$-electron system at the optimized geometry of the neutral molecule.

For an easy transfer of electrons, adsorption should occur on the portion of the molecule where the local softness, $S$, has the highest value. To describe site selectivity or local reactivity of atomic sites in a molecule, local reactivity descriptors (LRDs) have been proposed [25]. The LRDs, Fukui functions $(f(r))$ and the local softness $\left(s_{k}^{+}, s_{k}^{-}\right)$, within the framework of conceptual DFT have been utilized in this work. The values of $f(r)$ condensed to the $k^{\text {th }}$ atom in a molecule are calculated from Equations (7) and (8) proposed by Yang and Mortier [26] based on the finite difference approximations, from population analysis of atoms in molecules. In a given molecule, atoms with higher $f(r)$ values are potentially more reactive than those with lower $f(r)$ values.

$$
\begin{gathered}
f_{k}^{+}=q_{k}(N+1)-q_{k}(N) \\
f_{k}^{-}=q_{k}(N)-q_{k}(N-1)
\end{gathered}
$$

where $q_{k}(N), q_{k}(N-1)$ and $q_{k}(N+1)$ are respectively the electronic populations of the $k^{\text {th }}$ atom in the neutral, cationic and anionic species. Here, the geometries of the ionic species must be identical to that of the neutral molecule so that the external potential is maintained constant. The values of $f_{k}^{+}$and $f_{k}^{-}$ respectively describe the reactivity of atom $k$ toward nucleophiles (as an electrophile) and toward electrophiles (as a nucleophile) [26]. Local softness condensed to the $k^{\text {th }}$ atom in a molecule is calculated from their global values and the Fukui functions as follows [25] [27]: 


$$
s_{k}^{\alpha}=S f_{k}^{\alpha}
$$

with $\alpha=+$ or - , referring to nucleophilic or electrophilic respectively.

When two systems (for example a metal and an inhibitor) are brought together, electrons will flow from the system with lower electronegativity, $\chi$ (inhibitor) to that with higher $\chi$ (metal) until the chemical potentials become equal. In this work, the computed values of $\chi$ and $\eta$ have been used to predict the fraction of the electron transferred $(\Delta N)$ from the inhibitor to metallic surface as follows [28]:

$$
\Delta N=\frac{\chi_{\text {metal }}-\chi_{\text {inhibitor }}}{2\left(\eta_{\text {metal }}+\eta_{\text {inhibitor }}\right)}
$$

where $\chi_{\text {metal }}, \chi_{\text {inhibitor }}$ and $\eta_{\text {metal }}, \eta_{\text {inhibitor }}$ denote the absolute electronegativity and hardness of the species indicated as subscripts, respectively. The difference in electronegativity drives the electron transfer, and the sum of the hardness parameters acts as resistance [29]. In this study, $\eta_{\mathrm{Al}}=0$ while the theoretical value of the work function of bulk aluminium, $\phi_{\mathrm{Al}}=4.28 \mathrm{eV}$, were used in place of $\chi_{\mathrm{Al}}$ [30]. As such, Equation (10) can be rewritten thus:

$$
\Delta N=\frac{\phi_{\mathrm{Al}}-\chi_{\text {inhibitor }}}{2 \eta_{\text {inhibitor }}}
$$

Another important parameter calculated in this study is the initial inhibitor-metal interaction energy $(\Delta \psi)$, which has been introduced by Kokalj and Kovacevic [30]. The initial inhibitor-metal interaction energy $\Delta \psi$ has been calculated as follows:

$$
\Delta \psi=-\frac{\left(\chi_{\text {metal }}-\chi_{\text {inhibitor }}\right)^{2}}{4\left(\eta_{\text {metal }}+\eta_{\text {inhibitor }}\right)}
$$

which can equally be rewritten as follows:

$$
\Delta \psi=-\frac{\left(\chi_{\text {metal }}-\chi_{\text {inhibitor }}\right)^{2}}{4 \eta_{\text {inhibitor }}}
$$

where $\chi_{\text {metal }}, \chi_{\text {inhibitor }}$ and $\eta_{\text {metal }}, \eta_{\text {inhibitor }}$ denote the absolute electronegativity and hardness of the species indicated as subscripts, respectively.

To calculate the proton affinity $(P A)$ values of studied inhibitors, Equation (14) was employed:

$$
P A=E_{(\text {pro })}-\left(E_{(\text {non-pro) }}+E_{\mathrm{H}^{+}}\right)
$$

where, $E_{(\text {non-pro) }}$ and $E_{(\text {pro) }}$ are the corrected energies (including thermal and non-thermal corrections) of the non-protonated and protonated inhibitors respectively and $E_{\mathrm{H}^{+}}$is the corrected energy (including thermal and non-thermal corrections) of $\mathrm{H}^{+}$calculated thus:

$$
E_{\mathrm{H}^{+}}=E_{\mathrm{H}_{3} \mathrm{O}^{+}}-E_{\mathrm{H}_{2} \mathrm{O}}
$$

It is equally important to point out that the PA of a molecule is a measure of its basicity. 
Solvent effects were invoked implicitly via the conductor-like polarizable continuum model (CPCM) [31] along with the Gaussian charge scheme and making use of scaled van der Waals solute cavity surfaces as implemented in the ORCA 4.2.0 program. Also, the Solvation Model Density (SMD) method was used in the accurate calculation of solvation free energies.

\section{Results and Discussion}

\subsection{Molecular Descriptors of Anticorrosion Activity}

\subsubsection{Frontier Molecular Orbitals (FMOs)}

The FMOs (comprising the highest occupied molecular orbital (HOMO) and the lowest unoccupied molecular orbital (LUMO)) are the most important molecular orbitals used to study chemical trends. Although the commonly used Kohn-Sham DFT (KS-DFT) provides the best cost-accuracy ratio in the calculation of molecular orbital energies, it may fail to predict highly accurate molecular orbital energies [32]. This is particularly true for the LUMO eigenvalue and consequently the HOMO-LUMO gaps, reason being that KS-DFT is essentially a workhorse for ground state properties, by means of which the accurate computation of virtual LUMO orbitals is not guaranteed [32]. To circumvent this setback, the use of the time-dependent DFT (TD-DFT) method in conjunction with hybrid functionals to calculate more accurate LUMO eigenvalues (and subsequently more accurate HOMO-LUMO gaps) has been recommended [33]. Accordingly, the TD-DFT formalism has been used herein to compute accurate FMO energies and HOMO-LUMO gaps at the PBE0-D3/def2-TZVP//PBEh-3c level of theory. The PBE0 global hybrid functional has been employed here because it generally provides accurate estimates of the first excitation singles for systems with a predominance of local $n \rightarrow \pi^{*}$ and $\pi \rightarrow \pi^{*}$ transitions [34]. The FMO parameters of inhibitors calculated in this study include: the highest occupied molecular orbital energy $\left(E_{\text {Номо }}\right)$, the lowest unoccupied molecular orbital energy $\left(E_{\mathrm{LUMO}}\right)$, the energy gap ( $\left.\Delta E=E_{\text {номо }}-E_{\mathrm{LUMO}}\right)$ as shown in Table 1.

Based on the FMO theory, chemical reactivity is a function of the interaction between the HOMO and LUMO of the reacting species. $E_{\mathrm{HO} о}$ is usually linked to the capability of a chemical species to donate electron to neighboring molecules. Organic molecules with relatively less negative (or more positive) HOMO energy values are expected to have high electron donating abilities, leading to high corrosion inhibition efficiencies [5]. As can be observed from Table 1, the

Table 1. FMO properties of studied inhibitors calculated from single point energies at the TD-DFT-D3-PBE0/def2-TZVP level in gas phase.

\begin{tabular}{cccccc}
\hline \multirow{2}{*}{ Parameters } & \multicolumn{5}{c}{ Inhibitors } \\
\cline { 2 - 6 } & HL1 & HL2 & HL3 & HL4 & HL5 \\
\hline$E_{\text {HOMO }}(\mathrm{eV})$ & -6.180 & -6.336 & -6.013 & -6.023 & -6.207 \\
$E_{\mathrm{LUMO}}(\mathrm{eV})$ & -1.736 & -2.762 & -1.644 & -1.655 & -1.769 \\
$\Delta E(\mathrm{eV})$ & 4.444 & 3.574 & 4.369 & 4.368 & 4.438 \\
\hline
\end{tabular}


$E_{\mathrm{HOMO}}$ values vary according to the ranking: HL3 > HL4 > HL1 > HL5 > HL2, which reveals the predilection of HL3 to donate electrons to the incompletely filled $3 \mathrm{p}$ orbitals of the aluminium metal, as opposed to HL2 which has the least HOMO energy. It can equally be observed that the strongly deactivating nitro group in HL2 diminishes the electron donating ability of its HOMO, whereas the moderately activating alkoxy group in HL3 produces an opposite effect. Therefore, the adsorption of corrosion inhibitor molecules on the surface of aluminium could be improved upon by incorporating suitable functional site groups in the inhibitor.

Unlike the trend shown by the HOMO energies, the LUMO energies on their part have revealed the ability of the LUMOs to accept electrons from the filled $3 \mathrm{~s}$ orbital of aluminium, leading to a strong interaction energy. It is clear from $\mathrm{Ta}$ ble 1 that HL2 with the lowest LUMO value will readily accept electrons from the filled $3 \mathrm{~s}$ orbital of aluminium. It can equally be observed that HL3 bearing a moderately activating group is the least disposed to interact with the 3 s orbital of aluminium. The HOMO and LUMO isosurfaces of the inhibitors currently investigated are displayed in Table 2. As a general observation, the HOMO of each inhibitor molecule is largely localized around the imidazole moiety, which is indicative of the fact that the metal-HOMO interactions are mainly in the vicinity of the imidazole moiety. Contrary to the HOMOs, the LUMOs are almost entirely localized in the regions around the benzene ring $(\mathrm{A})$ and the unsaturated keto-alkene moiety. On a relative scale, the HL2 inhibitor best interacts with the aluminium metal through its 3 s orbital, given that $E_{\mathrm{LUMO}}$ value is highest.

The energy gap $(\Delta E)$ is widely accepted as a measure of the global reactivity of a molecule. Generally, the inhibition efficiency of an organic inhibitor is expected to be high for a molecule with low $\Delta E$ [35]. From the values of $\Delta E$, it is clear that the corrosion inhibition efficiency of the investigated inhibitors is ranked according to the order: HL2 > HL4 > HL3 > HL5 > HL1, which is quite consistent with the separate trends in both the HOMO and LUMO energies.

\subsubsection{Reactivity Descriptors}

The global reactivity parameters of the molecules studied: ionization energy (IP), electronic affinity $(E A)$, global electronegativity $(\chi)$, global hardness $(\eta)$, global softness $(S)$, fraction of electrons transferred $(\Delta N)$, initial inhibitor-metal interaction energy $(\Delta \psi)$, dipole moment, $\mu$, total energy $(T E)$ and electrophilicity index $(\omega)$, calculated based on the finite difference approximation, are listed in Table 3. The $E A$ values show the following trend: HL4 < HL1 < HL5 < HL3 < HL2, which suggests that HL2 loses the highest amount of energy when the $3 \mathrm{~s}$ orbital of the aluminium metal donates electrons to its LUMO, leading to a more stabilizing interaction between the metal and HL2 inhibitor. Indeed, the corrosion inhibition tendency shown by the $E A$ parameter is in good agreement with that trend earlier revealed by the LUMO energies. This trend can be explained by the fact that the inhibitors with deactivating groups (except HL3), tend to resist electron donation compared to inhibitors with activating groups. 
Table 2. The frontier molecular orbitals (FMOs) of studied inhibitors obtained at the TD-DFT-D3-PBE0/def2-TZVP//PBEh-3c levels in gas phase.

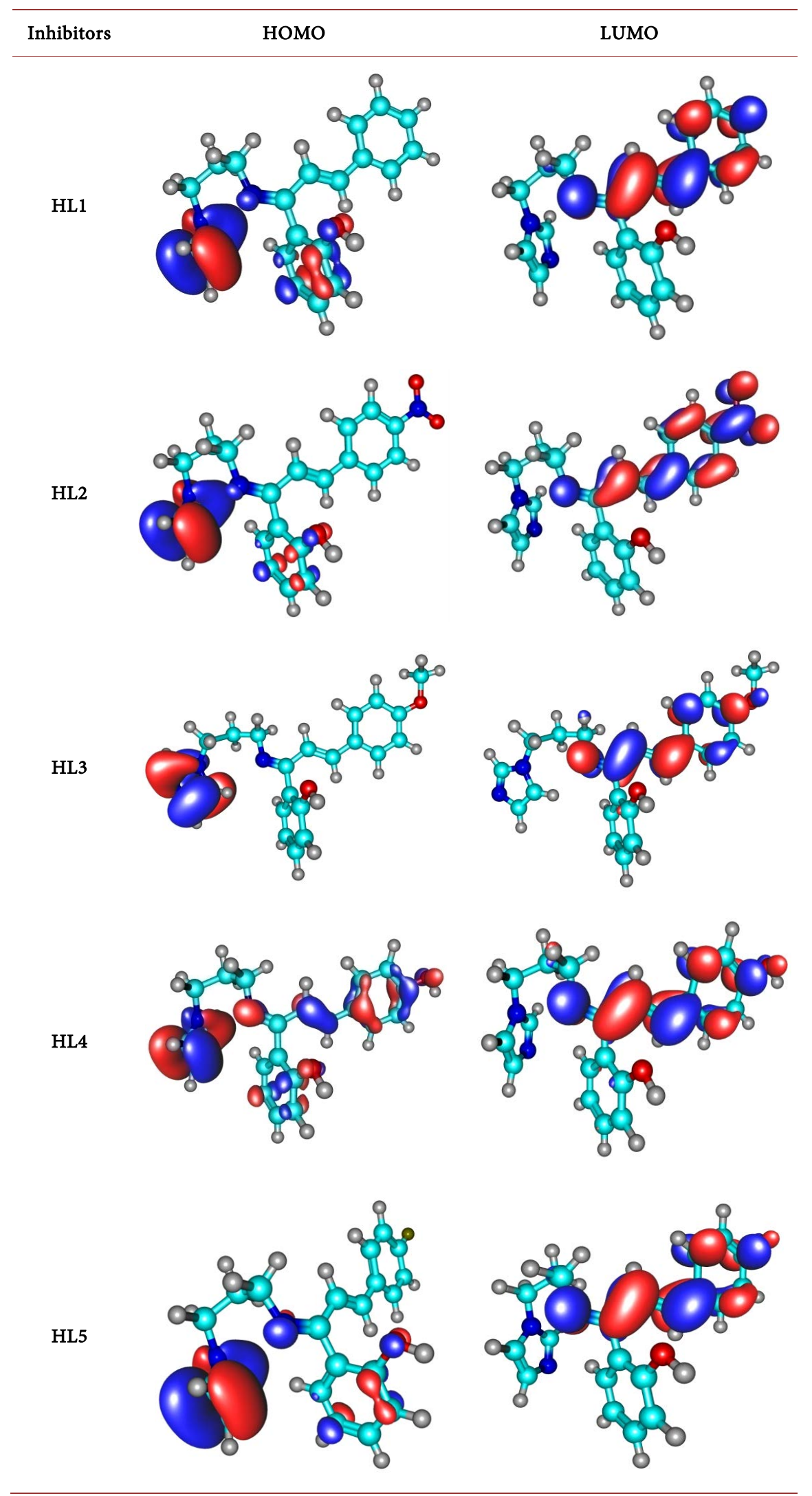


Table 3. Molecular properties of studied inhibitors calculated from single point energies at the PBE0/def2-TZVP//PBEh-3c levels in gas phase.

\begin{tabular}{cccccc}
\hline \multirow{2}{*}{ Parameters } & \multicolumn{5}{c}{ Inhibitors } \\
\cline { 2 - 6 } & $\mathrm{HL1}$ & $\mathrm{HL2}$ & HL3 & HL4 & HL5 \\
\hline$I P(\mathrm{eV})$ & 7.435 & 7.738 & 7.235 & 7.202 & 7.481 \\
$E A(\mathrm{eV})$ & 0.574 & 1.632 & 0.725 & 0.454 & 0.649 \\
$\chi(\mathrm{eV})$ & 4.004 & 4.685 & 3.980 & 3.828 & 4.065 \\
$\eta(\mathrm{eV})$ & 3.430 & 3.053 & 3.255 & 3.374 & 3.416 \\
$S(\mathrm{eV}-1)$ & 0.292 & 0.328 & 0.307 & 0.296 & 0.293 \\
$\Delta \boldsymbol{N}$ & 0.775 & -0.618 & 0.488 & 0.763 & 0.367 \\
$\Delta \psi$ & -0.00555 & -0.01343 & -0.00691 & -0.01514 & -0.00338 \\
$\mu(\mathrm{Debye})$ & 4.689 & 4.114 & 9.062 & 5.168 & 3.788 \\
$\omega(\mathrm{eV})$ & 27.504 & 33.499 & 25.778 & 24.717 & 28.218 \\
$T E(\mathrm{Eh})$ & -1049.600 & -1253.674 & -1163.847 & -1124.665 & -1148.661 \\
\hline
\end{tabular}

The influence of dipole moment on anticorrosion ability is controversial because some studies have shown that a small dipole moment is required for better inhibition, whereas others argue that a high value of the dipole moment enhances corrosion inhibition. The latter argument is based on the fact that the adsorption potency between the inhibitor and the metal surface increases with an increase in dipole moment [35]. In our opinion, the orientation of the dipole moment of an inhibitor molecule should contribute in fashioning its aggregation pattern over the metal surface through some dipole-dipole, dipole-charge interactions among other interactions [36], thus affecting the degree of surface coverage of the inhibitor. The orientation with respect to the Cartesian coordinate axes of the dipole moment vectors of the molecules currently studied are shown in Figure 2. Apparently, the dipole moment orientation of HL2 deviates significantly from the trend shown by the other inhibitors. This may be linked to the strongly deactivating nitro group in HL2.

The amount of electrons transferred $(\Delta N)$ have been routinely used to explain the tendency of a molecule to donate electrons; and as reported in [37], the higher the amount of electrons transferred, the higher the tendency of a compound to donate electrons to a neighboring chemical species that is electron-deficient. It is well-known that the higher the number of electrons transferred $(\Delta N)$, the higher the anticorrosion potency of an inhibitor. In fact, $\Delta N$ can be regarded as a derived descriptor from the electronegativity/hardness equalization principle. It was pointed out that positive values of $\Delta N$ indicate that the molecules act as electron donors meanwhile negative values indicate that the molecules act as electron acceptors [38]. Therefore, the $\Delta N$ values obtained for the currently investigated inhibitors have shown a higher inclination towards donating electrons to the metal surface. It is clear from Table 3 that the HL5 inhibitor has the highest tendency to transfer electrons to the aluminium metal. 

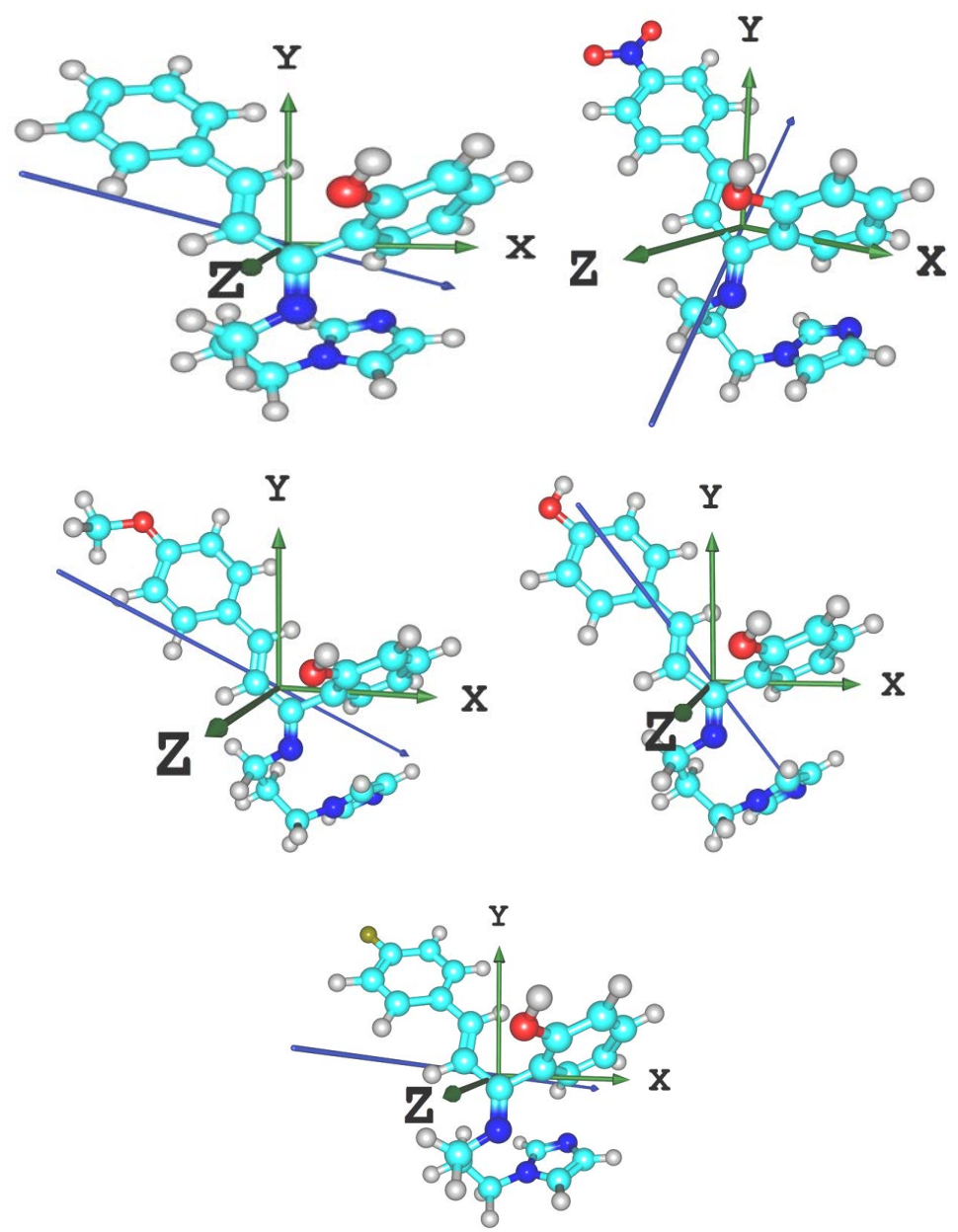

Figure 2. Optimized structures of inhibitors at PBEh-3c level of theory. The dipole moment vector is shown in blue and the coordinate axes in green.

This tendency decreases in the order: HL3 $>$ HL4 $>$ HL1 $>$ HL2. It can also be noticed that the HL2 molecule has a $\Delta N$ value which is negative indicative of its very low partiality to offer electrons to the metal. Finally, the trend in the initial inhibitor-metal interaction energy $(\Delta \psi)$ from Table 3 is as follows: HL4 $>$ HL2 $>$ HL3 > HL1 > HL5.

\subsection{Study of One of the Most Feasible Inhibitor-Aluminium Interaction Patterns}

As earlier mentioned, the formation of metallic complexes is one of the factors that affect the adsorption of an inhibitor onto the metal surface [3]. In a bid to simulate implicitly the interaction between inhibitor and metal, inhibitor-aluminium complexes were modelled and optimized without any constraints. Herein, the input chelates as shown in Figure 3 were modelled as a follow up of the bonding pattern proposed by Kalanithi and co-workers towards metal ions [39] and secondly, based on the HOMO isosurfaces localization pattern portrayed in Table 2, which shows the most plausible regions for the strongest metal-inhibitor interactions. 


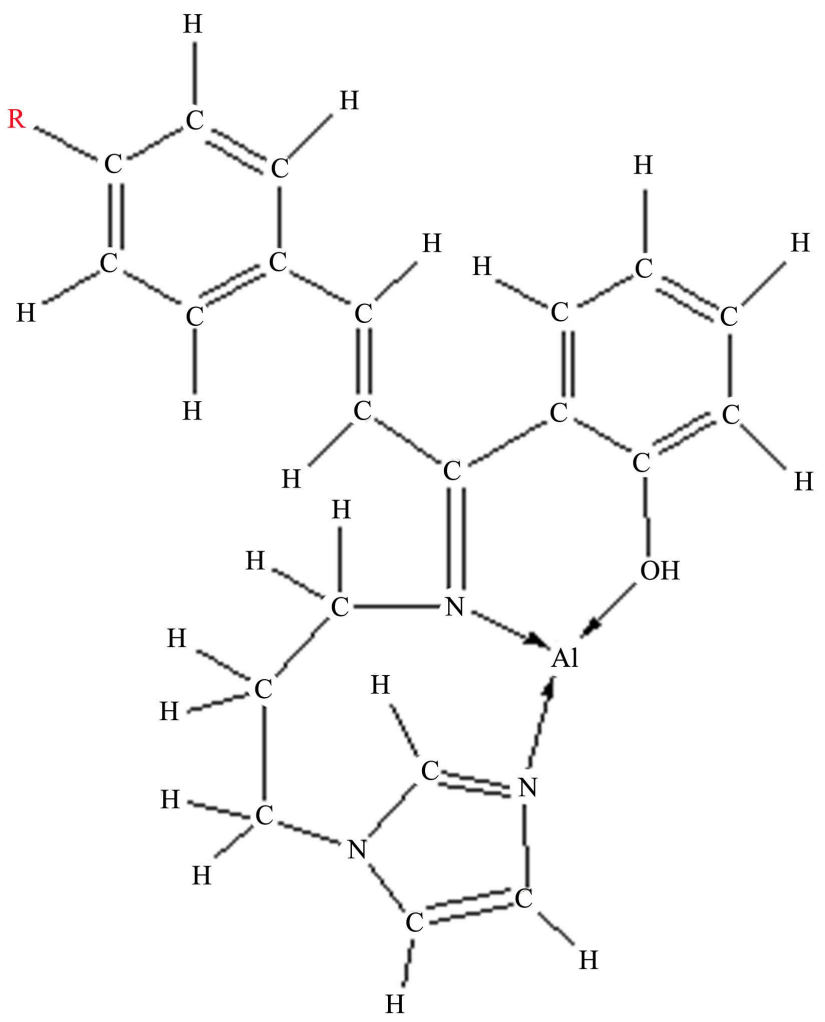

Figure 3. Input geometry of inhibitor-aluminium complex in gas phase.

Figure 4 shows the optimized complexes with a few selected bond lengths in atomic units. It is observed that a V-shaped geometry about the aluminium central metal is obtained in all studied complexes.

Also, the Al-O (25) and Al-N (10) bond lengths are observed to be longest in both the HL2-aluminium (2.111 a.u. and 1.906 a.u. respectively) and HL5-aluminium complexes (2.109 a.u. and 1.892 a.u. respectively) due to electron depletion around the coordination center by the nitro and fluoro deactivating groups in both HL2 and HL5. This electron depletion tendency renders the aforementioned bonds slightly weaker.

\subsubsection{Stability of Inhibitor-Aluminium Complex Interaction Based on Binding Energy}

The theoretical likelihood of inhibitors binding to the aluminium atom and the stability of the resulting complexes in gas phase were verified via the calculation of thermodynamic parameters such as the binding energies $\Delta E_{\text {binding }}$ ), according to the supramolecular approach, the enthalpies $\left(\Delta H_{\text {binding }}^{0}\right.$ ), and Gibbs free energies $\left(\Delta G_{\text {binding }}^{0}\right)$ at $298.15 \mathrm{~K}$ and $1.00 \mathrm{~atm}$, as expressed respectively in Equations (16), (17) and (18).

$$
\begin{gathered}
\Delta E_{\text {binding }}=E_{\text {complex }}-\left(E_{\mathrm{Al}}+E_{\text {inhibitor }}\right) \\
\Delta H_{\text {binding }}^{0}=H_{\text {complex }}^{0}-\left(H_{\mathrm{Al}}^{0}+H_{\text {inhibitor }}^{0}\right) \\
\Delta G_{\text {binding }}^{0}=G_{\text {complex }}^{0}-\left(G_{\mathrm{Al}}^{0}+G_{\text {inhibitor }}^{0}\right)
\end{gathered}
$$



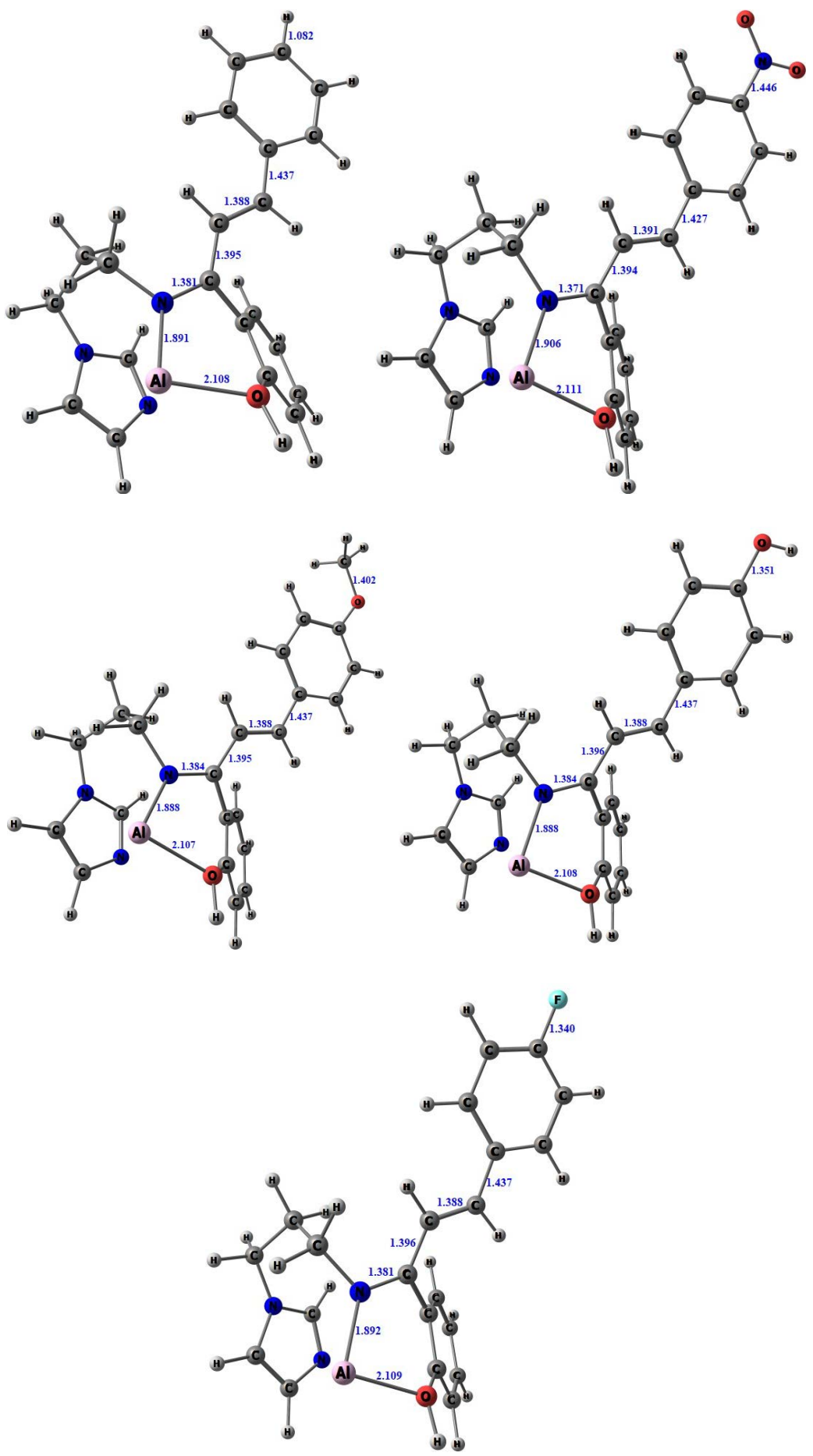

Figure 4. Optimized structures of the inhibitor-aluminium complexes showing some selected bond length values in a.u., using the PBEh-3c method in gas phase.

Herein, the calculated binding energies (as indicated in Table 4) have been corrected for basis set superposition errors (BSSE) using the geometrical counterpoise correction ( $\mathrm{gCP}$ ) approach [19] as implemented in the ORCA 4 package. The gas-phase molecular thermodynamic properties ( $\Delta H_{\text {binding }}^{0}$ and $\Delta G_{\text {binding }}^{0}$ ) have been computed using the KiSThelP program [40]. 
Table 4. Calculated values $\Delta E_{\text {binding }}(\mathrm{eV}), \Delta H_{\text {binding }}^{0}(\mathrm{eV}), \Delta G_{\text {binding }}^{0}(\mathrm{eV})$ of the Aluminium-inhibitor complexes at PBEh-3c level of theory in gas phase.

\begin{tabular}{cccccc}
\hline \multirow{2}{*}{$\begin{array}{c}\text { Thermodynamic } \\
\text { parameters }\end{array}$} & HL1-Al & HL2-Al & HL3-Al & HL4-Al & HL5-Al \\
\cline { 2 - 6 } & -2.742 & -2.883 & -2.689 & -2.687 & -2.749 \\
$\Delta E_{\text {binding }}$ & -2.690 & -2.831 & -2.637 & -2.636 & -2.698 \\
$\Delta H_{\text {binding }}^{0}$ & -2.118 & -2.252 & -2.057 & -2.089 & -2.108 \\
$\Delta G_{\text {binding }}^{0}$ & & & & \\
\hline
\end{tabular}

It is clear from Table 4 that the stabilities of these complexes are confirmed by their negative $\Delta E_{\text {binding }}(\mathrm{eV})$ values. This shows that their formation from the inhibitors and the aluminium metal is energetically favorable. The strongest binding energy is observed to occur in the HL2-aluminium complex while the HL4-aluminium complex is the weakest in the series.

This is supported by the fact that all binding enthalpies are negative, thus signifying that aluminium-inhibitor binding is exothermic in each case studied. Moreover, the Gibbs free energies for aluminium-inhibitor binding are all negative, implying that aluminium-inhibitor bindings are thermodynamically feasible. These results thus highlight the spontaneity of formation and stability of the complexes studied herein.

\subsubsection{Quantum Theory of Atoms-in-Molecules (QTAIM) Analysis of Some Bond Interactions within the Inhibitor-Aluminium Complexes}

In view of characterizing and revealing auxiliary inhibitor-aluminium bond interactions, Bader's QTAIM has been employed herein, based on topological parameters at bond critical points (BCP).

In topology analysis, the real space function, eta index $(\eta)$, given by $\eta=\frac{\left|\lambda_{1}(r)\right|}{\lambda_{3}(r)}$ where $\lambda_{1}$ and $\lambda_{3}$ are the lowest and the highest eigenvalues of hessian matrix of the electron density, $\rho$, respectively, has been argued to have values at bond critical point less than unity for closed shell interactions for such ionic and van der Waals interactions and increases with increasing covalent character [41]. Identified bond interaction in the framework of QTAIM based on the optimized inhibitor-aluminium complexes in gas phase at PBEh-3c level of theory are shown in the molecular maps in Figure 5. In the present study, topological analyses of additional real space functions such as the electron density and its laplacian have been performed at bond critical points and presented in Table 5. Large $\rho(r)$ values and $\nabla^{2} \rho(r)<0$ indicate polar and non-polar covalent bonding interactions, whereas small $\rho(r)$ values and $\nabla^{2} \rho(r)>0$ indicate closed-shell interactions [42]. Generally, $\rho(r)$ is greater than 0.20 a.u. for covalent bonding interactions and less than 0.10 a.u. for closed-shell interactions [42]. The values of $\nabla^{2} \rho(r)$ and $-G(r) / V(r)$ were also used to characterize the bonding interactions in the compounds studied. Generally, $G(r)$ is the kinetic 


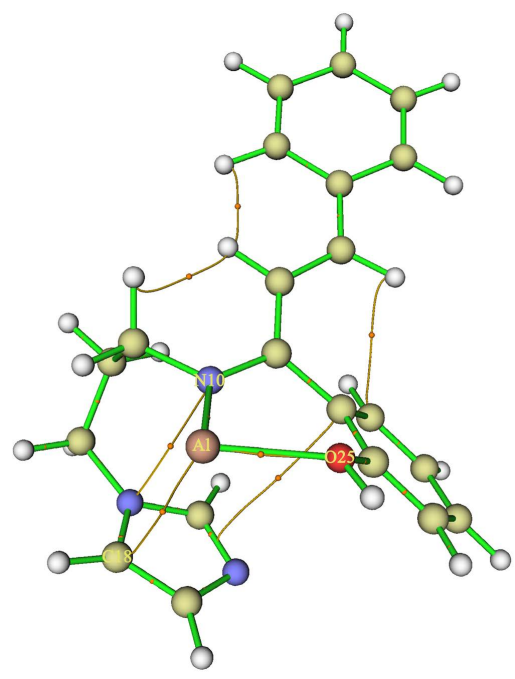

HL1

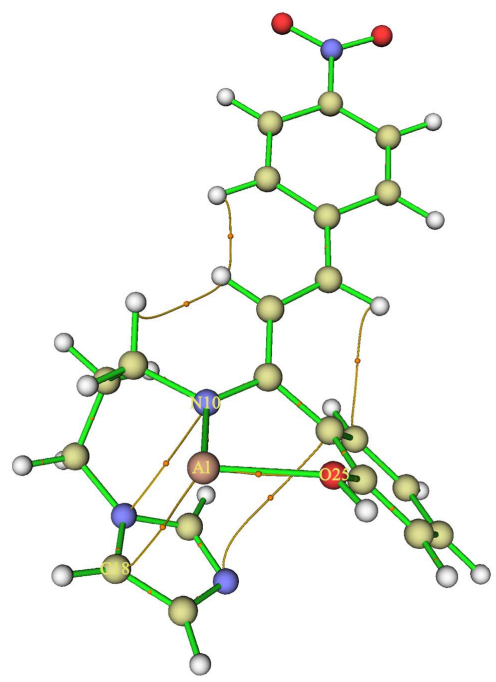

HL2

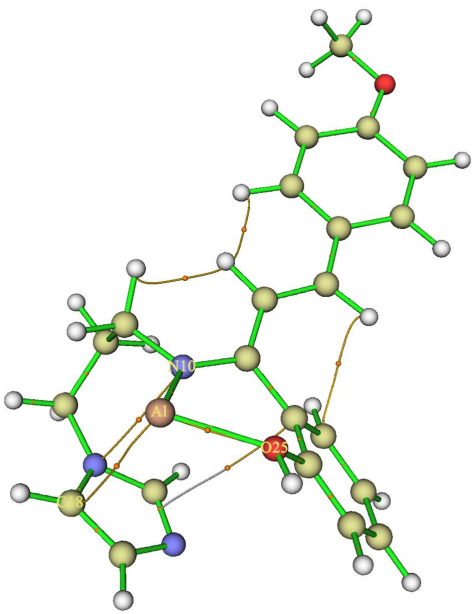

HL3

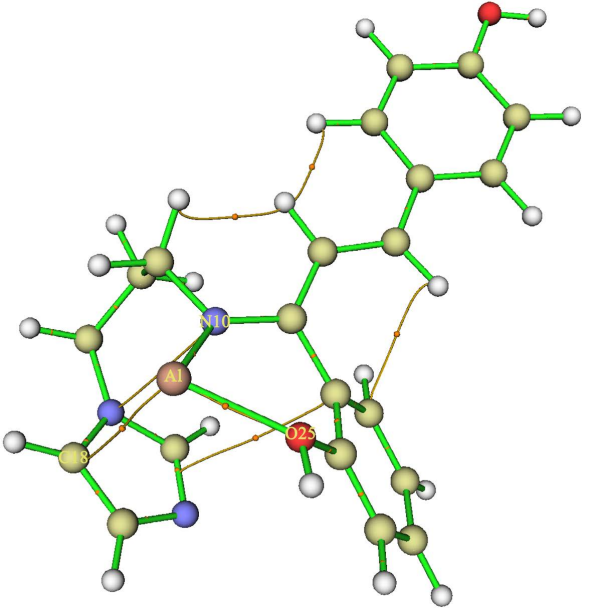

HL4

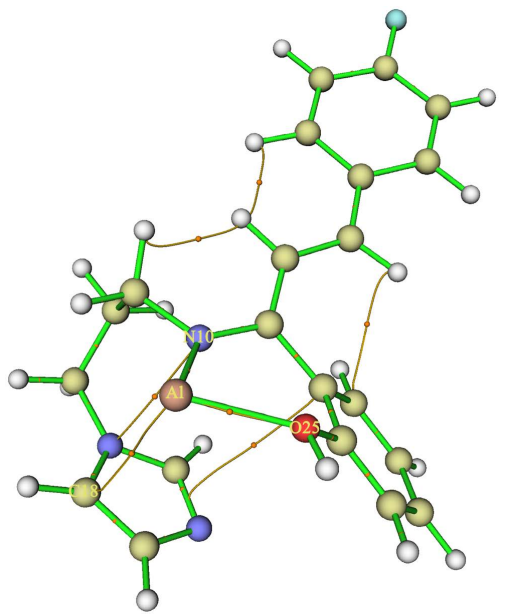

HL5

Figure 5. Molecular graphs of inhibitor-aluminium complexes based on their gas phase optimized geometries at the PBEh-3c level of theory in gas phase. The BCPs are the small red spheres around the center of the bond paths. The bond paths are the brown or green colored lines while aluminium atoms are the large brown spheres. 
Table 5. Bonding interactions and values of the electron density $[\rho(r)]$, the Laplacian $\left[\nabla^{2} \rho(r)\right]$, kinetic energy density $[G(r)]$, potential energy density $[V(r)]$, eta index, all measured in a.u., and interatomic interaction energy in $\mathrm{eV}\left(E_{\text {int }}\right)$ for the bonding interactions in the inhibitor-aluminium complexes studied in gas phase.

\begin{tabular}{|c|c|c|c|c|c|c|c|}
\hline \multirow{2}{*}{$\begin{array}{l}\text { Bonding } \\
\text { Interaction }\end{array}$} & \multicolumn{6}{|c|}{ Real Space Functions } & \multirow{2}{*}{$E_{\text {int }}(\mathrm{eV})$} \\
\hline & $\rho(r)$ & $\nabla^{2}(r)$ & $G(r)$ & $V(r)$ & $-G / V$ & eta index & \\
\hline \multicolumn{8}{|l|}{ HL1-Al } \\
\hline $\mathrm{Al} \cdots \mathrm{C}_{18}$ & 0.0121 & 0.0136 & 0.0041 & -0.0048 & 0.8508 & 0.2412 & -0.0659 \\
\hline $\mathrm{Al} \cdots \mathrm{N}_{10}$ & 0.0742 & 0.4571 & 0.1195 & -0.1247 & 0.9584 & 0.1744 & -1.6961 \\
\hline $\mathrm{Al} \cdots \mathrm{O}_{25}$ & 0.0372 & 0.1797 & 0.0455 & -0.0462 & 0.9866 & 0.1549 & -0.6280 \\
\hline \multicolumn{8}{|l|}{ HL2-Al } \\
\hline $\mathrm{Al} \cdots \mathrm{C}_{18}$ & 0.0128 & 0.0137 & 0.0043 & -0.0052 & 0.8331 & 0.2482 & -0.0701 \\
\hline $\mathrm{Al} \cdots \mathrm{N}_{10}$ & 0.0719 & 0.4334 & 0.1133 & -0.1182 & 0.9582 & 0.1748 & -1.6087 \\
\hline $\mathrm{Al} \cdots \mathrm{O}_{25}$ & 0.0372 & 0.1765 & 0.0450 & -0.0458 & 0.9818 & 0.1566 & -0.6232 \\
\hline \multicolumn{8}{|l|}{ HL3-Al } \\
\hline $\mathrm{Al} \cdots \mathrm{C}_{18}$ & 0.0120 & 0.0136 & 0.0041 & -0.0048 & 0.8545 & 0.2406 & -0.0651 \\
\hline $\mathrm{Al} \cdots \mathrm{N}_{10}$ & 0.0747 & 0.4631 & 0.1210 & -0.1263 & 0.9584 & 0.1743 & -1.7181 \\
\hline $\mathrm{Al} \cdots \mathrm{O}_{25}$ & 0.0372 & 0.1807 & 0.0457 & -0.0463 & 0.9880 & 0.1544 & -0.6299 \\
\hline \multicolumn{8}{|l|}{ HL4-Al } \\
\hline $\mathrm{Al} \cdots \mathrm{C}_{18}$ & 0.0120 & 0.0136 & 0.0041 & -0.0048 & 0.8539 & 0.2399 & -0.0653 \\
\hline $\mathrm{Al} \cdots \mathrm{N}_{10}$ & 0.0747 & 0.4632 & 0.1210 & -0.1263 & 0.9585 & 0.1743 & -1.7183 \\
\hline $\mathrm{Al} \cdots \mathrm{O}_{25}$ & 0.0371 & 0.1799 & 0.0456 & -0.0462 & 0.9873 & 0.1546 & -0.6280 \\
\hline \multicolumn{8}{|l|}{ HL5-Al } \\
\hline $\mathrm{Al} \cdots \mathrm{C}_{18}$ & 0.0124 & 0.0137 & 0.0042 & -0.0049 & 0.8455 & 0.2456 & -0.0673 \\
\hline $\mathrm{Al} \cdots \mathrm{N}_{10}$ & 0.0741 & 0.4564 & 0.1193 & -0.1244 & 0.9585 & 0.1744 & -1.6929 \\
\hline $\mathrm{Al} \cdots \mathrm{O}_{25}$ & 0.0371 & 0.1791 & 0.0454 & -0.0461 & 0.9861 & 0.1549 & -0.6267 \\
\hline
\end{tabular}

energy density at the BCP (always positive) and $V(r)$ is the potential energy density at the BCP (always negative) [43] When $\nabla^{2} \rho(r)>0$ and $-G(r) / V(r)>1$, the interactions are noncovalent, whereas when $\nabla^{2} \rho(r)>0$ and $0.5<-G(r) / V(r)<1$, the interactions are partially or partly covalent [44]. Based on the popular equation formulated by Espinosa [45] $\left(E_{\text {int }}=0.5 \mathrm{~V}(r)\right)$, for estimating interatomic noncovalent bond interactions, selected bond interaction energies were calculated accordingly.

A critical examination of Table 5 and Figure 5 shows that in addition to the $\mathrm{Al}-\mathrm{O}$ (25) and Al-N (10) bonds earlier portrayed in the optimized complexes in Figure 4, there exists an Al-C (18) bond interaction in all studied complexes. From the eta, $\rho(r),-G(r) / V(r)$ and $\nabla^{2} \rho(r)$ values, it can be concluded that all selected bond interactions in Table 4 are closed shell interactions with partly covalent character. This partial covalent character is more pronounced for the $\mathrm{Al}-\mathrm{O}$ (25) and Al-N (10) bond interactions. Based on the interaction energy val- 
ues, it is obvious that the Al-N (10) bond interactions are the strongest with energies ranging between $-1.6087 \mathrm{eV}$ in the $\mathrm{HL} 2-\mathrm{Al}$ complex to $-1.7183 \mathrm{eV}$ in $\mathrm{HL} 4-\mathrm{Al}$, followed by the $\mathrm{Al}-\mathrm{O}$ (25) bond interactions with energies ranging from $-0.6232 \mathrm{eV}$ in the HL2-Al to $-0.6299 \mathrm{eV}$ in the HL3-Al complex. Finally, the $\mathrm{Al}-\mathrm{C}$ (18) are the weakest with values ranging between $-0.0651 \mathrm{eV}$ in HL3-Al and $-0.0701 \mathrm{eV}$ in HL2-Al. These interaction energies between inhibitor and aluminium metal from QTAIM analysis corroborate results earlier observed in the binding energy thermodynamics parameter. Indeed, all the complexes modeled have been found to be relatively stable.

\subsection{Local Energy Decomposition (LED) Analysis of the Inhibitor-Aluminium Complexes}

In a bid to obtain more insight into the various types of contributions to the non-covalent interactions at the interface of the inhibitor fragment (fragment 1 ) and the aluminium atom fragment (fragment 2), the domain-based local pair natural orbital CCSD (T) method, i.e., DLPNO-CCSD (T), was used in calculating the interaction energy between the two fragments, albeit at a relative chemical accuracy. Decomposition of the interaction energy has been done via the local energy decomposition (LED) [22] scheme. In this study, the open-shell variant of the DLPNO-CCSD (T)/LED scheme [21] was employed. It should be recalled that the DLPNO-CCSD (T)/LED method takes into account counterpoise correction thus compensating for basis set incompleteness effects [22]. For exploratory and a more or less qualitative decomposition of the interaction energy, some chemically meaningful components of the interaction energy are presented in Table 6.

In Table 6, $E_{\text {exch }}^{\text {ref }}$ represents the inter-fragment exchange interaction, $E_{\text {elstat }}^{\text {ref }}$ is the electrostatic energy interaction between the distorted electronic clouds of the fragments, $E_{\text {disp }}^{\mathrm{C} C \mathrm{CSD}}$ term contains the London dispersion contribution from the strong pairs described above plus the interfragment component of the weak pairs, which is essentially dispersive in nature, $E_{\mathrm{C}-\mathrm{SP}}^{\mathrm{CT}(1 \rightarrow 2)}$ and $E_{\mathrm{C}-\mathrm{SP}}^{\mathrm{CT}(2 \rightarrow 1)}$ are the dynamic polarization terms (i.e. charge-transfer components at the correlated level) and the ratio of the dispersion energy $E_{\text {disp }}^{\text {C-CSD }}$ to the entire interaction energy $\Delta E_{\text {int }},\left(\frac{\Delta E_{\text {disp }}^{\text {C-CSD }}}{\Delta E_{\text {int }}}\right)$, gives the character of different interactions with

Table 6. Summary of some selected contributions (energies in Hatrees) of the LED at the DLPNO-CCSD (T) level of theory in gas phase.

\begin{tabular}{|c|c|c|c|c|c|c|c|}
\hline Molecules & $E_{\text {elstat }}^{\text {ref }}$ & $E_{\text {exch }}^{\mathrm{ref}}$ & $E_{\text {disp }}^{\text {C.CCSD }}$ & $E_{\mathrm{C}-\mathrm{SP}}^{\mathrm{CT}(1 \rightarrow 2)}$ & $E_{\mathrm{C}-\mathrm{SP}}^{\mathrm{CT}(2 \rightarrow 1)}$ & $\Delta E_{\mathrm{int}}$ & $\Delta E_{\mathrm{disp}}^{\mathrm{C}-\mathrm{CCSD}} / \Delta E_{\mathrm{int}}$ \\
\hline $\mathrm{HL} 1 \cdots \mathrm{Al}$ & -1.3407 & -0.0884 & -0.0117 & -0.0497 & -0.0084 & -1.4565 & 0.0080 \\
\hline $\mathrm{HL} 2 \cdots \mathrm{Al}$ & -1.2968 & -0.0868 & -0.0119 & -0.0483 & -0.0084 & -1.4113 & 0.0084 \\
\hline $\mathrm{HL} 3 \cdots \mathrm{Al}$ & -1.3476 & -0.0887 & -0.0116 & -0.0500 & -0.0083 & -1.4636 & 0.0079 \\
\hline $\mathrm{HL} 4 \cdots \mathrm{Al}$ & -1.3465 & -0.0886 & -0.0116 & -0.0499 & -0.0083 & -1.4625 & 0.0079 \\
\hline HL5 $\cdots \mathrm{Al}$ & -1.3355 & -0.0884 & -0.0117 & -0.0498 & -0.0084 & -1.4515 & 0.0081 \\
\hline
\end{tabular}


respect to the importance of dispersion. Hence, a ratio exceeding 1 unambiguously identifies a dispersion dominated interaction. Furthermore, a ratio between 0.5 and 1 points to an interaction with balanced contributions of dispersive and nondispersive characters, while a ratio smaller than 0.5 suggests an interaction somewhat dominated by nondispersive contributions [22]. It is evident from Table 6 therefore that, the character of the interaction between the inhibitors and aluminium metal are largely nondispersive in nature. Also, it is clear that charge transfer between fragments is dominant from fragment 1 to 2 , with some back donation from fragment 2 to 1 . As a result, net-charge flow is found to be towards the metal. As anticipated, electrostatic energy interactions are the most contributing to the binding of inhibitors on to the aluminium metal. The interaction energies show that the interactions in all complexes are attractive with slight variations in strength. Accordingly, HL3 $\cdots \mathrm{Al}$ complex is the most attractive at the calculated coupled-cluster level.

\subsection{Local Parameters of Studied Anticorrosion Inhibitors}

In order to verify the probable role played by each atom in the molecules studied towards anticorrosion, their local reactivity parameters (Fukui functions (FF)) $f_{k}^{+}$and $f_{k}^{-}$, and local softness $s_{k}^{+}$and $s_{k}^{-}$based on Hirshfeld charges (Hirshfeld population analysis) have been calculated. It should be indicated that the Hirshfeld population scheme has been used here because it is not basis set dependent and give intuitively positive FF values [46]. However, due to orbital relaxation effects, some negative FF values can still be observed [47]. It is observed from Figure 6 that the sites acting favorably for nucleophilic attacks are
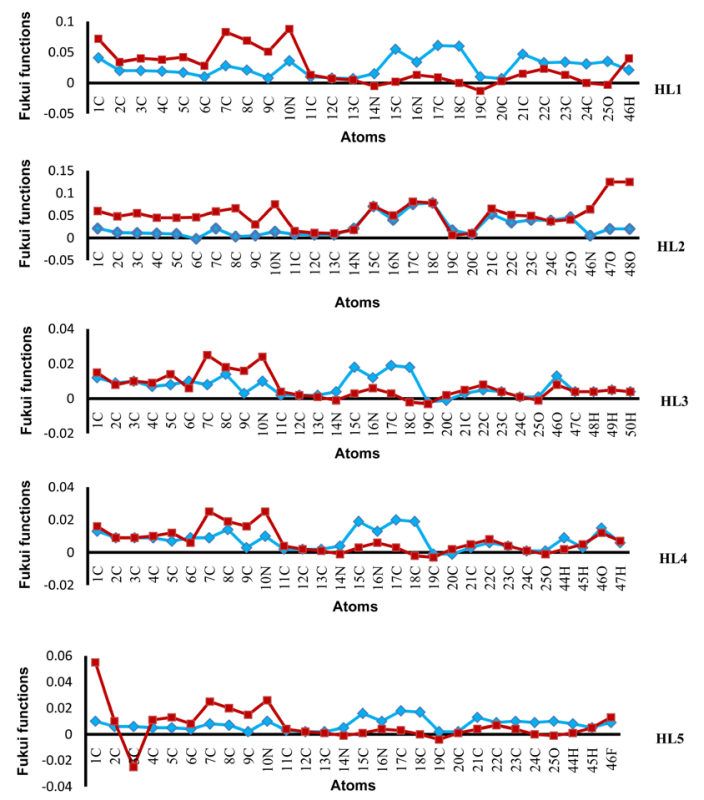

$\underset{\rightarrow-\text { condensed fukui function for electrophilic attack on atom } \mathrm{k}}{\rightarrow}$

Figure 6. Variation of condensed Fukui functions $\left(f_{k}^{+}\right.$and $\left.f_{k}^{-}\right)$for the studied inhibitors (all values in a.u.) on atom $\mathrm{k}$. 
typically the carbon atoms of the imidazole ring (15C, 17C and 18C). Given that these sites are equally observed to be the softest to nucleophilic attack, they make use of their HOMO orbitals to donate electrons to the incompletely filled $3 p$ orbital of the aluminium metal. These results support the observed localization of HOMO orbitals over the imidazole ring of the inhibitors shown earlier in Table 2. On the other hand, the most prominent sites acting favorably for electrophilic attacks are typically the atoms $10 \mathrm{~N}, 7 \mathrm{C}, 8 \mathrm{C}$ and $9 \mathrm{C}$ in all the molecules, in addition to the $47 \mathrm{O}$ and $48 \mathrm{O}$ in the HL2 molecule. These atoms therefore are responsible for interaction with the metal surface by accepting electrons into the LUMO of the inhibitor. It can thus be predicted that in the HL2 molecule for example interaction between the molecule and the metal surface not only occurs around the imidazole, $10 \mathrm{~N}$ and $25 \mathrm{O}$ regions but also with the nitro group.

\subsection{Molecular Properties of Protonated Inhibitors in Acidified Water}

In acidic aqueous medium, corrosion inhibitors act as Lewis basis, as they accept protons from the acids in solution which otherwise interact with the metal surfaces, ionizing the metal surface and resulting in their degradation. Thus, corrosion inhibitors with strong proton affinity (PA) (more negative values of PA) are required to circumvent the effect of acid media on metals by capturing protons in these solutions. Thus, a strong PA value of a molecule increases its anticorrosion efficiency. In this study, the protonated forms of the studied molecules $\left(\mathrm{H}_{2} \mathrm{~L}^{+}, \mathrm{H}_{2} \mathrm{~L}^{+}, \mathrm{H}_{2} \mathrm{~L}^{+}, \mathrm{H}_{2} \mathrm{~L}^{+}\right.$and $\left.\mathrm{H}_{2} \mathrm{~L}^{+}\right)$in aqueous medium were studied at the PBEh-3c level of theory.

The most favorable computationally tested protonation site which brought about the lowest molecular energy in all studied inhibitors was found to be at the $10 \mathrm{~N}$ atom (see Figure 1) (after geometry optimization in water), corroborating the values of the condensed fukui functions for electrophilic attack $\left(f_{k}^{-}\right)$(see Figure 6) wherein one of the most favorable sites for electrophilic attacks is the $10 \mathrm{~N}$ atom. It is therefore evident that in acidic aqueous medium, the $10 \mathrm{~N}$ atom will be unavailable for any substantial interaction with the aluminium surface. According to proton affinity values of the studied inhibitors calculated at the PBEh-3c level of theory, the inhibition efficiency ranking for studied inhibitors is: HL3 > HL5 > HL4 > HL1 > HL2. This trend can be explained from the fact that the electron depleting groups tend to reduce the proton affinities while donor groups improve on the proton affinity power of the studied molecules. Interestingly, Figure 7 reveals the fact that the PAs (inhibition efficacy in acid medium) of the HL1 and HL3 are found to be directly proportional to temperature, within the studied temperature range 273.15 - $343.15 \mathrm{~K}$, whereas the HL2, HL4 and HL5 inhibitors show proton affinities that are inversely proportional to temperature within the studied temperature range, albeit with very slight changes in PAs. Consequently, at relatively higher temperatures in aqueous medium, the HL1 and HL3 are found to be more effective anticorrosion inhibitors 


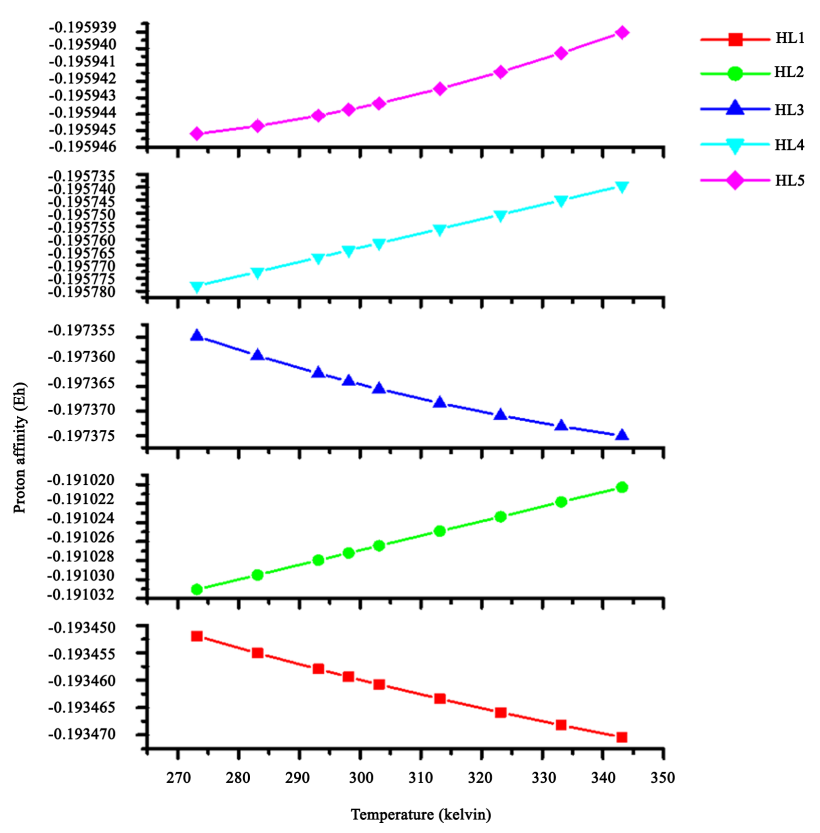

Figure 7. Proton affinity (PA) variation with temperature of studied inhibitors in aqueous medium.

than at lower temperatures, in contrast to the HL2, HL4 and HL5 compounds that will be better corrosion inhibitors at relatively higher temperatures.

Given that the energy gap in the protonated species is lower than unprotonated species in gas phase (see Table 1 and Table 6), it is can be predicted that the atoms of the imidazole moiety and the $9 \mathrm{C}, 8 \mathrm{C}, 7 \mathrm{C}$ and $6 \mathrm{C}$ of the chalcone moiety will be involved in the interaction with the aluminium surface. This improved interaction is confirmed by the slight increase in planarity of the 9C-8C-7C-6C moiety of the protonated molecules.

The calculated dihedral angle $\phi(9 \mathrm{C}-8 \mathrm{C}-7 \mathrm{C}-6 \mathrm{C})$ in $\mathrm{H}_{2} \mathrm{~L}^{+}$is found to be $179.3^{\circ}$ whereas in the HL1 molecule it is $178.9^{\circ}$. It must be stressed that high degree of planarity, that may lead to a high electronic delocalization are typical of a resonant system [37]. This indicates that the molecular interaction with the metallic surface can also occur through the $\pi$ orbitals of the chalconic moiety in aqueous phase. The same observation is obtained for $\mathrm{H}_{2} \mathrm{~L}^{+}$and its HL5 counterpart wherein the $\phi$ (9C-8C-7C-6C) dihedral angle is respectively $179.7^{\circ}$ and $179.1^{\circ}$.

However, the planarity of the $9 \mathrm{C}-8 \mathrm{C}-7 \mathrm{C}-6 \mathrm{C}$ portion of the $\mathrm{H}_{2} \mathrm{~L}^{+}$molecule is found to lower compared to the HL4 counterpart. The dihedral angles $\phi$ (9C-8C-7C-6C) here are found to be $179.3^{\circ}$ and $179.7^{\circ}$, showing that the interaction of this portion of the molecule with the aluminium surface drops upon protonation in acidic aqueous medium. In acidic aqueous medium also, studies have shown that the energy gaps of the protonated species presented in Table 7 witness a decrease compared to the values in the unprotonated species in the gas phase reminiscence of an even improved adsorption in acidic aqueous medium of the unprotonated species in gas phase. 
Table 7. Molecular properties of studied protonated inhibitors in acidic aqueous medium calculated from single point energies at the TD-DFT-D3-PBE0/def2-TZVP//PBEh-3c levels in aqueous medium.

\begin{tabular}{cccccc}
\hline \multirow{2}{*}{ Parameters } & \multicolumn{5}{c}{ Protonated Inhibitors } \\
\cline { 2 - 6 } & $\mathrm{H}_{2} \mathrm{L1}^{+}$ & $\mathrm{H}_{2} \mathrm{L2}^{+}$ & $\mathrm{H}_{2} \mathrm{~L}^{+}$ & $\mathrm{H}_{2} \mathrm{~L}^{+}$ & $\mathrm{H}_{2} \mathrm{~L}^{+}$ \\
\hline$E_{\text {Hомо }}(\mathrm{eV})$ & -6.906 & -6.762 & -6.595 & -6.649 & -6.865 \\
$E_{\mathrm{LUMO}}(\mathrm{eV})$ & -2.949 & -3.320 & -2.803 & -2.799 & -2.937 \\
$\Delta E(\mathrm{eV})$ & 3.957 & 3.442 & 3.792 & 3.850 & 3.928 \\
\hline
\end{tabular}

\subsection{Aqueous Absolute Standard One-Electron Redox Potentials of Studied Inhibitors $\left(\operatorname{Inh}_{s}\right)$}

In a bid to gain more knowledge on how well the inhibitors ( $\operatorname{Inh}_{s}=$ HL1, HL2, HL3, HL4 and HL5) under study bequeath electrons in aqueous solution phase, their one-electron redox process was investigated according to Scheme 1, and were used to determine their standard absolute redox potentials $\left(E_{\text {calc }}^{\circ}\right)$ accordingly.

Herein, the absolute redox potentials have been computed from the Gibbs free energy associated with the oxidation-reduction process of the inhibitors in aqueous solution phase, by considering Equation (19):

$$
E_{\text {calc }}^{\circ}=\frac{\Delta G_{\text {solv }}^{\circ \text {,redox }}}{n F}
$$

where $n$ is the number of transferred electrons and $F$ is the Faraday constant ( $F$ $\left.=23.06 \mathrm{kcal} \cdot \mathrm{mol}^{-1} \cdot \mathrm{V}^{-1}\right)$. The $\Delta G_{\text {solv }}^{\circ}$,red in $\mathrm{kcal} \cdot \mathrm{mol}^{-1}$ is obtained by calculating the change in free energy in the gas phase, $\Delta G_{\text {gas }}^{\circ}$,rex , and the solvation free energies of each of the species, $\Delta G_{\text {solv }} \mathrm{Inh}^{+\cdot}$ for the oxidized species and $\Delta G_{\text {solv }}$ Inh for the reduced species. The overall balance equation is as follows:

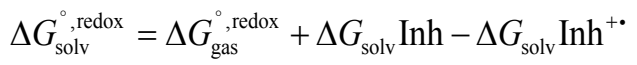

It should be noted that, the free energy of the electron is not taken into account since the addition of another half-cell reaction for the reference electrode automatically cancels the energy of the electron out. It could also be argued that the ionic convention of an electron leads to a zero value of its free energy in the gas and solution phase, hence it is typically ignored [48].

Within the framework of the SMD method, the standard-state solvation free energies, $\Delta G_{\text {solv }}^{\mathrm{o}}$, are calculated as the sum of three terms [49] [50]:

$$
\Delta G_{\text {solv }}^{\mathrm{o}}=\Delta G_{\mathrm{ENP}}+\Delta G_{\mathrm{CDS}}+\Delta G_{\mathrm{conc}}^{\mathrm{o}}
$$

In the ORCA program, the term $\Delta G_{\mathrm{ENP}}$ represents the bulk electrostatic contribution arising from the interaction of the medium and the molecular surface charges while the cavity-dispersion solvent-structure term ( $\left.\Delta G_{\mathrm{CDS}}\right)$ represents the free energy change associated with the solute-solvent interactions in the first solvation shell [50]. The term $\Delta G_{\text {conc }}^{\mathrm{o}}$ arises when changing from gas-phase at $1 \mathrm{~atm}$ and $298.15 \mathrm{~K}$ to solution phase at $1 \mathrm{~mol} \cdot \mathrm{L}^{-1}$ $\left(\Delta G_{\mathrm{conc}}^{\mathrm{o}}=1.89 \mathrm{kcal} \cdot \mathrm{mol}^{-1}\right)[49]$. 


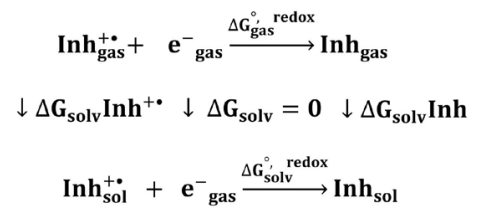

Scheme 1. Thermodynamic cycle for the calculation of Gibbs free energies of a one-electron redox process of inhibitors under study.

From Table 8, it can be noticed that the electron withdrawing groups show higher redox potentials which thus diminishes their affinity for aluminium in aqueous solution, which can influence their chelating ability for aluminium. Accordingly, the absolute redox potential ranking is as follows: HL2 > HL4 > HL5 > HL1 > HL3. It is thus expected that the HL3 will bequeath electrons in aqueous solution phase most easily, corroborating finding made on their $E_{\text {Номо }}$ studies which reveals the predilection of HL3 to donate electrons most. As expected, the HL2 redox couple has the least negative absolute redox potential suggestive of its resistance to bequeath electrons easily in aqueous medium. As already mentioned, the electron withdrawing/electron donating effect should be highly relevant for donor-acceptor interactions in the resulting inhibitor-aluminium complexes, since these are typically electrostatic in nature.

Table 8. Aqueous absolute standard one-electron redox potentials $\left(E_{\text {calc }}^{\circ}\right)$ in volts of studied corrosion inhibitors obtained at the DFT-D3BJ-PBE0/def2-TZVP//PBEh-3c levels.

\begin{tabular}{cccccc}
\hline Inh & $\Delta G_{\text {gas }}^{\circ \text {,redox }}$ & $\Delta G_{\text {solv }} \mathrm{Inh}^{+}$ & $\Delta G_{\text {solv }}$ Inh & $\Delta G_{\text {solv }}^{\circ \text {,redox }}$ & $E_{\text {calc }}^{\circ}(\mathrm{V})$ \\
\hline HL1 & -152.540 & -52.911 & -21.152 & -120.781 & -5.238 \\
HL2 & -159.517 & -62.324 & -22.391 & -97.193 & -5.186 \\
HL3 & -149.164 & -26.967 & -20.146 & -142.342 & -6.173 \\
HL4 & -151.888 & -56.266 & -24.045 & -119.667 & -5.189 \\
HL5 & -153.308 & -53.924 & -20.704 & -120.089 & -5.208 \\
\hline
\end{tabular}

${ }^{\star}$ All $\Delta \mathrm{G}$ values are measured in $\mathrm{kcal} \cdot \mathrm{mol}^{-1}$.

\section{Conclusion}

Quantum chemical calculations have been undertaken to evaluate the anticorrosion efficacy and modes of action of five chalcone-based imidazole ligands on aluminium in both gas phase and acidic aqueous medium. Computed values of the frontier molecular energy gaps in gas phase are in the range $3.574 \mathrm{eV}$ and $4.444 \mathrm{eV}$, which are found to be lower than similar values in the literature, suggestive of very good adsorptive and inhibition capacity [5]. In order to model the inhibitor-aluminium interaction patterns, the geometries of inhibitor-aluminium complexes were fully optimized. All calculated interaction energies and Gibbs free energies at $298.15 \mathrm{~K}$ and $1.00 \mathrm{~atm}$, were found to be negative, which shows that the formation of the investigated inhibitor-aluminium complexes is energetically favorable. QTAIM analysis on these complexes in gas phase further re- 
vealed the existence of Al-O (25), Al-N (10) and Al-C (18) bonding interactions. Additionally, LED analyses confirmed that, inhibitor-metal interactions in all investigated complexes are predominantly electrostatic in character and nondispersive in nature. Also, the proton affinity values in aqueous medium of the inhibitors investigated have revealed the following inhibition efficiency ranking: HL3 > HL5 > HL4 > HL1 > HL2. It has been found that for HL1 and HL3, the PA values are directly proportional to temperature within the range 273.15 $343.15 \mathrm{~K}$, whereas for HL2, HL4 and HL5, the PA values are nearly inversely proportional to temperature. In conclusion, our results have shown that the chalcones derivatives investigated in this work possess great potentials towards aluminium corrosion inhibition in both gas and acidic media.

\section{Acknowledgements}

This work has been supported by the Research Modernization grant to J.N. Ghogomu by the Cameroon Ministry of Higher Education and the Research Unit of Noxious Chemistry and Environmental Engineering (RUNOCHEE) of the University of Dschang-Cameroon, which are gratefully acknowledged.

\section{Conflicts of Interest}

The authors declare no conflicts of interest regarding the publication of this paper.

\section{References}

[1] Rani, B.E.A. and Basu, B.B.J. (2012) Green Inhibitors for Corrosion Protection of Metals and Alloys: An Overview. International Journal of Corrosion, 2012, Article ID: 380217. https://doi.org/10.1155/2012/380217

[2] Fang, J. and Li, J. (2002) Quantum Chemistry Study on the Relationship between Molecular Structure and Corrosion Inhibition Efficiency of Amides. Journal of Molecular Structure, 593, 179-185. https://doi.org/10.1016/S0166-1280(02)00316-0

[3] Salim, M.K., Ghassab, M.A. and Nozha, M.A. (2016) DFT Calculations on Corrosion Inhibition of Aluminium by Some Carbohydrates. International Journal of Biochemical Research Reviews, 14, 1-7. https://doi.org/10.9734/IJBCRR/2016/29288

[4] Kolawole, O.A. and Banjo, S. (2018) Theoretical Studies of Anti-Corrosion Properties of Triphenylimidazole Derivatives in Corrosion Inhibition of Carbon Steel in Acidic Media via DFT Approach. Analytical and Bioanalytical Electrochemistry, 10 136-146.

[5] Beda, R.H.B., Niamien, P.M., Bilé, E.B.A. and Trokourey, A. (2017) Inhibition of Aluminium Corrosion in $1.0 \mathrm{M} \mathrm{HCl}$ by Caffeine: Experimental and DFT Studies. Advances in Chemistry, 2017, Article ID: 6975248. https://doi.org/10.1155/2017/6975248

[6] Singh, P., Anand, A. and Kumar, V. (2014) Recent Developments in Biological Activities of Chalcones: A Mini Review. European Journal of Medicinal Chemistry, 85, 758-777. https://doi.org/10.1016/j.ejmech.2014.08.033

[7] Sirsat, S.B., Halikar, N.K., Pund, M.M. and Vartale, S.P. (2012) Synthesis and Biological Screening of Some Novel Hetero-Aryl Chalcone and Their Complexes. Re- 
search Journal of Pharmaceutical, Biological and Chemical Sciences, 3, 240-248.

[8] Quraishi, M.A., Chauhan, D.S. and Saji, V.S. (2020) Computational Methods of Inhibitor Evaluation. In: Quraishi, M.A., Chauhan, D.S. and Saji, V.S., Eds., Heterocyclic Organic Corrosion Inhibitors, Elsevier, Amsterdam, 59-86.

https://doi.org/10.1016/B978-0-12-818558-2.00003-5

[9] Obot, I.B., Haruna, K. and Saleh, T.A. (2018) Atomistic Simulation: A Unique and Powerful Computational Tool for Corrosion Inhibition Research. Arabian Journal of Science and Engineering, 44, 1-32. https://doi.org/10.1007/s13369-018-3605-4

[10] Xu, B., Yang, W.Z., Liu, Y., Yin, X.S., Gong, W.N. and Chen, Y.Z. (2014) Experimental and Theoretical Evaluation of Two Pyridinecarboxaldehyde Thiosemicarbazone Compounds as Corrosion Inhibitors for Mild Steel in Hydrochloric Acid Solution. Corrosion Science, 78, 260-268. https://doi.org/10.1016/j.corsci.2013.10.007

[11] Zhang, Z., Tian, N.C., Zhang, W.N., Huang, X.D., Ruan, L. and Wu, L. (2016) Inhibition of Carbon Steel Corrosion in Phase-Change Materials Solution by Methionine and Proline. Corrosion Science, 111, 675-689.

https://doi.org/10.1016/j.corsci.2016.06.005

[12] Singh, P., Ebenso, E.E., Olasunkanmi, L.O., Obot, I. and Quraishi, M. (2016) Electrochemical, Theoretical, and Surface Morphological Studies of Corrosion Inhibition Effect of Green Naphthyridine Derivatives on Mild Steel in Hydrochloric Acid. Journal of Physical. Chemistry C, 120, 3408-3419. https://doi.org/10.1021/acs.jpcc.5b11901

[13] Wang, D.P., Yang, D., Zhang, D.Q., Li, K., Gao, L.X. and Lin, T. (2015) Electrochemical and DFT Studies of Quinoline Derivatives on Corrosion Inhibition of AA5052 Aluminium Alloy in NaCl Solution. Applied Surface Science, 357, 2176-2183. https://doi.org/10.1016/j.apsusc.2015.09.206

[14] Singh, A., Ansari, K.R., Haque, J., Dohare, P., Lgaz, H., Salghi, R., et al. (2018) Effect of Electron Donating Functional Groups on Corrosion Inhibition of Mild Steel in Hydrochloric Acid: Experimental and Quantum Chemical Study. Journal of Taiwan Institute of Chemical Engineers, 82, 233-251. https://doi.org/10.1016/j.jtice.2017.09.021

[15] Obot, I.B., Ebenso, E.E. and Dao, D.Q. (2018) Editorial: Discovery of Novel Molecules for Corrosion Protection Using Computational Chemistry. Frontiers in Chemistry, 6, 277. https://doi.org/10.3389/fchem.2018.00277

[16] Neese, F. (2012) The ORCA Program System. Wiley Interdisciplinary Reviews. Computational Molecular Science, 2, 73-78. https://doi.org/10.1002/wcms.81

[17] Hanwell, M.D., Curtis, D.E., Lonie, D.C., Vandermeersch, T., Zurek, E. and Hutchison, G.R. (2012) Avogadro: An Advanced Semantic Chemical Editor, Visualization, and Analysis Platform. Journal of Cheminformatics, 4, Article ID: 17. https://doi.org/10.1186/1758-2946-4-17

[18] Grimme, S., Brandenburg, J.G., Bannwarth, C. and Hansen, A. (2015) Consistent Structures and Interactions by Density Functional Theory with Small Atomic Orbital Basis Sets. Journal of Chemical Physics, 143, Article ID: 054107. https://doi.org/10.1063/1.4927476

[19] Kruse, H. and Grimme, S. (2012) A Geometrical Correction for the Inter- and Intra-Molecular Basis Set Superposition Error in Hartree-Fock and Density Functional Theory Calculations for Large Systems. Journal of Chemical Physics, 136, Article ID: 154101. https://doi.org/10.1063/1.3700154

[20] Grimme, S., Ehrlich, S. and Goerigk, L. (2011) Effect of the Damping Function in 
Dispersion Corrected Density Functional Theory. Journal of Computational Chemistry, 32, 1456-1465. https://doi.org/10.1002/jcc.21759

[21] Altun, A., Saitow, M., Neese, F. and Bistoni, G. (2019) Local Energy Decomposition of Open-Shell Molecular Systems in the Domain-Based Local Pair Natural Orbital Coupled Cluster Framework. Journal of Chemical Theory and Computation, 15, 1616-1632. https://doi.org/10.1021/acs.jctc.8b01145

[22] Schneider, W.B., Bistoni, G., Sparta, M., Saitow, M., Riplinger, C., Auer, A.A. and Neese, F. (2016) Decomposition of Intermolecular Interaction Energies within the Local Pair Natural Orbital Coupled Cluster Framework. Journal of Chemical Theory and Computation, 12, 4778-4792. https://doi.org/10.1021/acs.jctc.6b00523

[23] Neese, F. (2003) An Improvement of the Resolution of the Identity Approximation for the Calculation of the Coulomb Matrix. Journal of Computational Chemistry, 24, 1740-1747. https://doi.org/10.1002/jcc.10318

[24] Neese, F. (2009) Prediction of Molecular Properties and Molecular Spectroscopy with Density Functional Theory: From Fundamental Theory to Exchange-Coupling. Coordination Chemistry Reviews, 253, 526-563.

https://doi.org/10.1016/j.ccr.2008.05.014

[25] Chattaraj, P.K., Giri, S. and Duley, S. (2011) Update 2 of: Electrophilicity Index. Chemical Reviews, 111, PR43-PR75. https://doi.org/10.1021/cr100149p

[26] Yang, W. and Mortier, W.J. (1986) The Use of Global and Local Molecular Parameters for the Analysis of the Gas-Phase Basicity of Amines. Journal of the American Chemical Society, 108, 5708-5711. https://doi.org/10.1021/ja00279a008

[27] Pérez, P., Domingo, L.R. and Aizman, A. (2007) The Electrophilicity Index in Organic Chemistry. In: Toro-Labbe, A., Ed., Theoretical Aspects of Chemical Reactivity, Vol. 19, Elsevier, Amsterdam, 139-201. https://doi.org/10.1016/S1380-7323(07)80010-0

[28] Lukovit, I., Kalman, E. and Zucchi, F. (2001) Corrosion Inhibitors-Correlation between Electronic Structure and Efficiency. Corrosion, 57, 3-8. https://doi.org/10.5006/1.3290328

[29] Lesar, A. and Milošev, I. (2009) Density Functional Study of the Corrosion Inhibition Properties of 1,2,4-triazole and Its Amino Derivatives. Chemical Physics Letters, 483, 198-203. https://doi.org/10.1016/j.cplett.2009.10.082

[30] Kokalj, A. and Kovacevic, N. (2011) On the Consistent Use of Electrophilicity Index and HSAB-Based Electron Transfer and Its Associated Change of Energy Parameters. Chemical Physics Letters, 507, 181-184. https://doi.org/10.1016/j.cplett.2011.03.045

[31] Barone, V. and Cossi, M. (1998) Quantum Calculation of Molecular Energies and Energy Gradients in Solution by a Conductor Solvent Model. Journal of Physical Chemistry A, 102, 1995-2001. https://doi.org/10.1021/jp9716997

[32] Sangalli, D., Ferretti, A., Miranda, H., Attaccalite, C., Marri, I., Cannuccia, E., Melo, P., Marsili, M., Paleari, F., Marrazzo, A., Prandini, G., Bonfà, P., Atambo, M.O., Affinito, M. P., Molina-Sánchez, A., Hogan, C., Grüning, M., Varsano, D. and Marini, A.F. (2019) Many-Body Perturbation Theory Calculations Using the Yambo Code. Journal of Physics. Condensed Matter, 31, Article ID: 325902. https://doi.org/10.1088/1361-648X/ab15d0

[33] Zhang, G. and Musgrave, C.B. (2007) Comparison of DFT Methods for Molecular Orbital Eigenvalue Calculations. Journal of Physical Chemistry A, 111, 1554-1561. https://doi.org/10.1021/jp061633o

[34] Adamo, C. and Jacquemin, D. (2013) The Calculations of Excited-State Properties 
with Time-Dependent Density Functional Theory. Chemical Society Reviews, 42, 845-856. https://doi.org/10.1039/C2CS35394F

[35] Costa, D., Ribeiro, T., Cornette, P. and Marcus, P. (2016) DFT Modeling of Corrosion Inhibition by Organic Molecules: Carboxylates as Inhibitors of Aluminium Corrosion. Journal of Physical Chemistry C, 120, 28607-28616. https://doi.org/10.1021/acs.jpcc.6b09578

[36] Lien, E.J., Guo, Z.R., Li, R.R. and Su, C.T. (1982) Use of Dipole Moment as a Parameter in Drug-Receptor Interaction and Quantitative Structure-Activity Relationship Studies. Journal of Pharmaceutical Science, 71, 641-655.

https://doi.org/10.1002/jps.2600710611

[37] Gomez, B., Likhanova, N.V., Dominguez-Aguilar, M.A., Martınez-Palou, R., Vela, A. and Gazquez, J.L. (2006) Quantum Chemical Study of the Inhibitive Properties of 2-Pyridyl-Azoles. Journal of Physical Chemistry B, 110, 8928-8934. https://doi.org/10.1021/jp057143y

[38] Guo, L., Safi, Z.S., Kaya, S., Shi, W., Tüzün, B., Altunay, N. and Kaya C. (2018) Anticorrosive Effects of Some Thiophene Derivatives Against the Corrosion of Iron: A Computational Study. Frontiers in Chemistry, 6, 155. https://doi.org/10.3389/fchem.2018.00155

[39] Kalanithi, M., Rajarajan, M., Tharmaraj, P. and Sheela, C.D. (2012) Spectral, Biological Screening of Metal Chelates of Chalcone Based Schiff Bases of N-(3aminopropyl) Imidazole. Spectrochimica Acta Part A, 87, 155-162. https://doi.org/10.1016/j.saa.2011.11.031

[40] Canneaux, S., Bohr, F. and Henon, E. (2014) KiSThelP: A Program to Predict Thermodynamic Properties and Rate Constants from Quantum Chemistry Results. Journal of Computational Chemistry, 35, 82-93. https://doi.org/10.1002/jcc.23470

[41] Niepötter, B., Herbst-Irmer, R., Kratzert, D., Prinson, P.S., Mondal, K.C., Roesky, H.W., Jerabek, P., Frenking, G. and Stalke, D. (2014) Experimental Charge Density Study of a Silylone. Angewandte Chemie International Edition, 53, 2766-2770. https://doi.org/10.1002/anie.201308609

[42] Nkungli, K.N. and Ghogomu, N.J. (2017) Theoretical Analysis of the Binding of iron (III) Protoporphyrin IX to 4 Methoxyacetophenone Thiosemicarbazone via DFT-D3, MEP, QTAIM, NCI, ELF, and LOL Studies. Journal of Molecular Modeling, 23, Article ID: 200. https://doi.org/10.1007/s00894-017-3370-4

[43] Cremer, D. and Kraka, E. (1985) Theoretical Determination of Molecular Structure and Conformation. 15. Three-Membered Rings: Bent Bonds, Ring Strain, and Surface Delocalization. Journal of the American Chemical Society, 107, 3800-3810. https://doi.org/10.1021/ja00299a009

[44] Bayat, M., Yaghoobi, F., Salehzadeh, S. and Hokmi, S. (2011) A Theoretical Study on the Interaction of $\left[\mathrm{Al}\left(\mathrm{H}_{2} \mathrm{O}\right)_{6}\right]^{3+}$ and $\left[\mathrm{Mg}\left(\mathrm{H}_{2} \mathrm{O}\right)_{6}\right]^{2+}$ Cations with Fullerene $\left(\mathrm{C}_{60}\right)$, Coronene and Benzene $\pi$-Systems. Polyhedron, 30, 2809-2814. https://doi.org/10.1016/j.poly.2011.08.017

[45] Espinosa, E., Molins, E. and Lecomte, C. (1998) Hydrogen Bond Strength Revealed by Topological Analyses of Experimentally Observed Electron Densities. Chemical Physics Letters, 285, 170-173. https://doi.org/10.1016/S0009-2614(98)00036-0

[46] Hirshfeld, F.L. (1977) Bonded-Atom Fragments for Describing Molecular Charge Densities. Theoretica Chimica Acta, 44, 129-138. https://doi.org/10.1007/BF00549096

[47] Melin, J., Ayers, P.W. and Ortiz, J.V. (2007) Removing Electrons Can Increase the Electron Density: A Computational Study of Negative Fukui Functions. Journal of 
Physical Chemistry A, 111, 10017-10019. https://doi.org/10.1021/jp075573d

[48] Bartmess, J.E. (1994) Thermodynamics of the Electron and the Proton. Journal of Physical Chemistry, 98, 6420-6424. https://doi.org/10.1021/j100076a029

[49] Marenich, A.V., Cramer, C.J. and Truhlar, D.G. (2009) Universal Solvation Model Based on Solute Electron Density and on a Continuum Model of the Solvent Defined by the Bulk Dielectric Constant and Atomic Surface Tensions. Journal of Physical Chemistry B, 113, 6378-6396. https://doi.org/10.1021/jp810292n

[50] Guan, D., Lui, R. and Matthews, S. (2020) LogP Prediction Performance with the SMD Solvation Model and the M06 Density Functional Family for SAMPL6 Blind Prediction Challenge Molecules. Journal of Computer-Aided Molecular Design, 34, 511-522. https://doi.org/10.1007/s10822-020-00278-1 